\title{
Complementary and Alternative Exercises for Management of Osteoarthritis
}

\author{
Ming-Chien Chyu, ${ }^{1,2,3,4}$ Vera von Bergen, ${ }^{4}$ Jean-Michel Brismée, ${ }^{5}$ Yan Zhang, ${ }^{6,7}$ \\ James K. Yeh, ${ }^{8}$ and Chwan-Li Shen ${ }^{4,7}$ \\ ${ }^{1}$ Graduate Healthcare Engineering, Whitacre College of Engineering, Texas Tech University, Lubbock, TX 79409, USA \\ ${ }^{2}$ Department of Mechanical Engineering, Whitacre College of Engineering, Texas Tech University, Lubbock, TX 79409, USA \\ ${ }^{3}$ Department of Health, Exercise, and Sport Sciences, Texas Tech University, Lubbock, TX 79409, USA \\ ${ }^{4}$ Department of Pathology, Texas Tech University Health Sciences Center, BB 198, 3601 th street, Lubbock, TX 79430-9097, USA \\ ${ }^{5}$ Center for Rehabilitation Research, Texas Tech University Health Sciences Center, Lubbock, TX 79430, USA \\ ${ }^{6}$ Community and Family Medicine, Texas Tech University Health Sciences Center, Lubbock, TX 79430, USA \\ ${ }^{7}$ Laura W. Bush Institute for Women's Health, Texas Tech University Health Sciences Center, Lubbock, TX 79430, USA \\ ${ }^{8}$ Applied Core Laboratory, Winthrop-University Hospital, New York, Mineola, NY 11501, USA
}

Correspondence should be addressed to Chwan-Li Shen, leslie.shen@ttuhsc.edu

Received 25 January 2011; Revised 28 March 2011; Accepted 3 May 2011

Academic Editor: Rana Hinman

Copyright ( $) 2011$ Ming-Chien Chyu et al. This is an open access article distributed under the Creative Commons Attribution License, which permits unrestricted use, distribution, and reproduction in any medium, provided the original work is properly cited.

\begin{abstract}
Osteoarthritis (OA) is a chronic condition characterized by degeneration of cartilage and its underlying bone within a joint. With no cure currently available, the goals of treating OA are to alleviate pain, maintain, or improve joint mobility, increase the muscle strength of the joints, and minimize the disabling effects of the disease. Recent research has suggested that complementary and alternative medicine (CAM) exercises may improve OA symptoms. This paper covers CAM mind-body exercises-Tai Chi, qigong, and yoga-for OA management and evaluates their benefits in pain reduction, muscle strength, physical function, stiffness, balance, fear of falling, self-efficacy, quality of life, and psychological outcomes in patients with OA, based on randomized controlled trials published. Findings from the literature suggest that CAM exercises demonstrate considerable promise in the management of OA. Future studies require rigorous randomized controlled trials with larger sample sizes.
\end{abstract}

\section{Introduction}

Osteoarthritis (OA), the most common joint disorder, is a major cause of disability in the aging population with its prevalence increasing and consequences significantly impacting society [1]. As the population ages worldwide, OA has become a serious health threat to many countries [2]. It is estimated that almost $18 \%$ of women and $10 \%$ of men 60 years of age and older have symptomatic OA [3]. Eighty percent of those with OA report limitations in movement, while $25 \%$ report inability to perform major daily activities of life [3]. The economic costs associated with OA sequelae have increased tremendously in the past decade and are predicted to continue to grow [4].
Pain, muscle weakness, and physical dysfunction form a vicious cycle in the knee OA, with muscle weakness being associated with pain and physical dysfunction [5]. Individuals with $\mathrm{OA}$ can experience difficulty in walking, balance deficit, and muscle weakness, thus increasing the risk of falling by threefold [6] and consequently, fallrelated fractures [7]. Well-established modifiable risk factors of OA include overweight, injury, occupation, structural malalignment, and muscle weakness, while nonmodifiable risk factors include older age, female gender, race, and genetic predisposition [2].

There is no cure for OA, as it is very difficult to restore the cartilage [8]. The goals of treatment are to alleviate pain, maintain, or improve joint mobility, increase the muscle 
strength of the joints, and minimize the disabling effects of the disease [9-13]. Nonpharmacological intervention such as exercise has been emphasized by the medical community [14]. Recent research has suggested that complementary and alternative medicine (CAM) exercises may improve OA symptoms [12, 13].

The use of CAM modalities for chronic musculoskeletal disorders, such as $\mathrm{OA}$, is primarily to alleviate associated discomfort and disability, and approximately $50 \%$ of patients with chronic musculoskeletal disorders reported using CAM modalities [9].

Among different CAM modalities, mind-body therapy is one of the most popular domains in the US [15]. Mindbody exercises can be broken down into subsections of tai chi (TC), qigong, and yoga in the mind-body therapy category covering a wide range of healing practices that share a common intention to improve the mind's capacity to affect bodily function and symptoms [15]. Although significant attention has focused on stretching and strengthening the quadriceps muscles for reducing symptoms of knee OA [1620], numerous studies have been conducted investigating effects of CAM exercise on OA. The aim of this study is to review the evidence provided by published randomized clinical trials (RCTs) for the effect of TC, qigong, or yoga on various clinical and quality of life outcomes among people with OA.

\section{Tai Chi for OA}

According to the 2007 National Health Interview Survey (NHIS), an estimated 2.2 million people in the US practice TC for their health [21]. TC is a moderate-intensity mindbody exercise with breath control training to promote vital energy (Qi) and blood circulation [22, 23]. It is a more holistic oriental life cultivation program than a simple physical exercise from a Western point of view. TC features gentle, smooth, graceful, coordinated, and flowing movements of different body parts, emphasizing constant shifting of body weight between two legs with both knees flexed all the time while meditating and breathing deeply [24].

People practice TC for various health purposes such as improving physical condition, muscle strength, coordination, flexibility, and balance, decreasing risk for falls, pain, stiffness, and fatigue, and improving sleep, cardiovascular and respiratory function, and overall wellness [25-29]. Tai chi involves slowly stretching the limbs and trunk, requires less physical strength than strenuous exercise, and can be suitable for physically frail older adults to practice in a small space, at any time, individually or in groups, regardless of weather conditions [30]. The National Arthritis Foundation has begun to promote a TC program to improve the quality of life for people with arthritis. A recent systematic review concluded that exercise programs based on TC seems to demonstrated better outcomes in functional aerobic capacity (ability to perform activities of daily living that require sustained aerobic metabolism) than hydrotherapy programs [31]. Another recent systematic review from the same authors concluded that exercise programs based on TC demonstrated better outcomes than mixed exercise programs based primarily on aerobic resistance, strength, and flexibility training in reducing pain in adults with lower limb OA [32].

Among all the mind-body exercises in CAM, TC is by far the most studied for the management of OA. Table 1 summarizes results of all RCT studies, 8 altogether, available in the open literature investigating TC's effects on OA management. All these RCTs are small, with total sample sizes ranging from only 16 participants to 97 participants. RCTs not published in peer-reviewed journals [33] or with too small sample size ( $<10$ people per allocation) [34] are excluded from the present study.

Various styles of TC were employed for intervention in these studies, including Chen, Yang, Wu/Hao, and Sun styles. Among different TC styles, the "Sun" style is the most studied although it is not the most popular style among TC practitioners worldwide. According to Song et al. [35], Sunstyle TC is characterized by slow and continuous movements with follow-up steps and higher stance with bending knees less than other types of TC. This style includes agile steps and exercises that may improve mobility, breathing, and relaxation. The movements do not require deep bending or squatting, which makes it easier and more comfortable to learn [35]. In fact, the characteristics of Sun style TC described above are quite common among all other styles of TC, and most TC can be practiced in high or low stance as the practitioner desires.

2.1. Pain Reduction. Pain is the predominant symptom of OA. Seven RCTs on TC's effects on pain reduction in OA patients have been published [24, 33-40]. Five of the those RCTs reported that TC significantly reduces pain intensity in OA patients, as measured by the Western Ontario and McMaster Universities osteoarthritis index (WOMAC) pain subscale, visual analogue scale (VAS), or pain intensity in SF36 pain subscale survey, compared to control [40], attention control $[26,39]$, and usual physical activity [35, 39] (Table 1). However, on the other hand, 2 studies reported that TC provided no benefits in pain reduction with arthritis pain self-efficacy assessment compared to waiting list control [36], and attention control [37]. Both of these 2 studies reporting no benefits by TC in pain reduction involved patients with $\mathrm{OA}$ at locations other than the knee, while all those studies focusing only on patients with knee OA demonstrated TC's benefits in pain reduction. This suggests that the slow, gentle, and weight-carrying TC footwork provides pain-relieving effects on OA at the knee, a body part strongly emphasized and required in TC training. Such pain-relieving effect is not significant or even absent at other body parts, particularly those in the upper body, where less weight-carrying activity than knee is involved in TC. TC was also found to result in no difference compared with hydrotherapy in pain reduction in OA patients [36]. However, hydrotherapy requires a major facility (pool), while TC intervention does not need major facility or equipment and can even be implemented at home [24].

The discrepancy of TC's impact on pain reduction in OA patients among different studies cannot be explained by their differences in sample size, intervention duration, frequency 


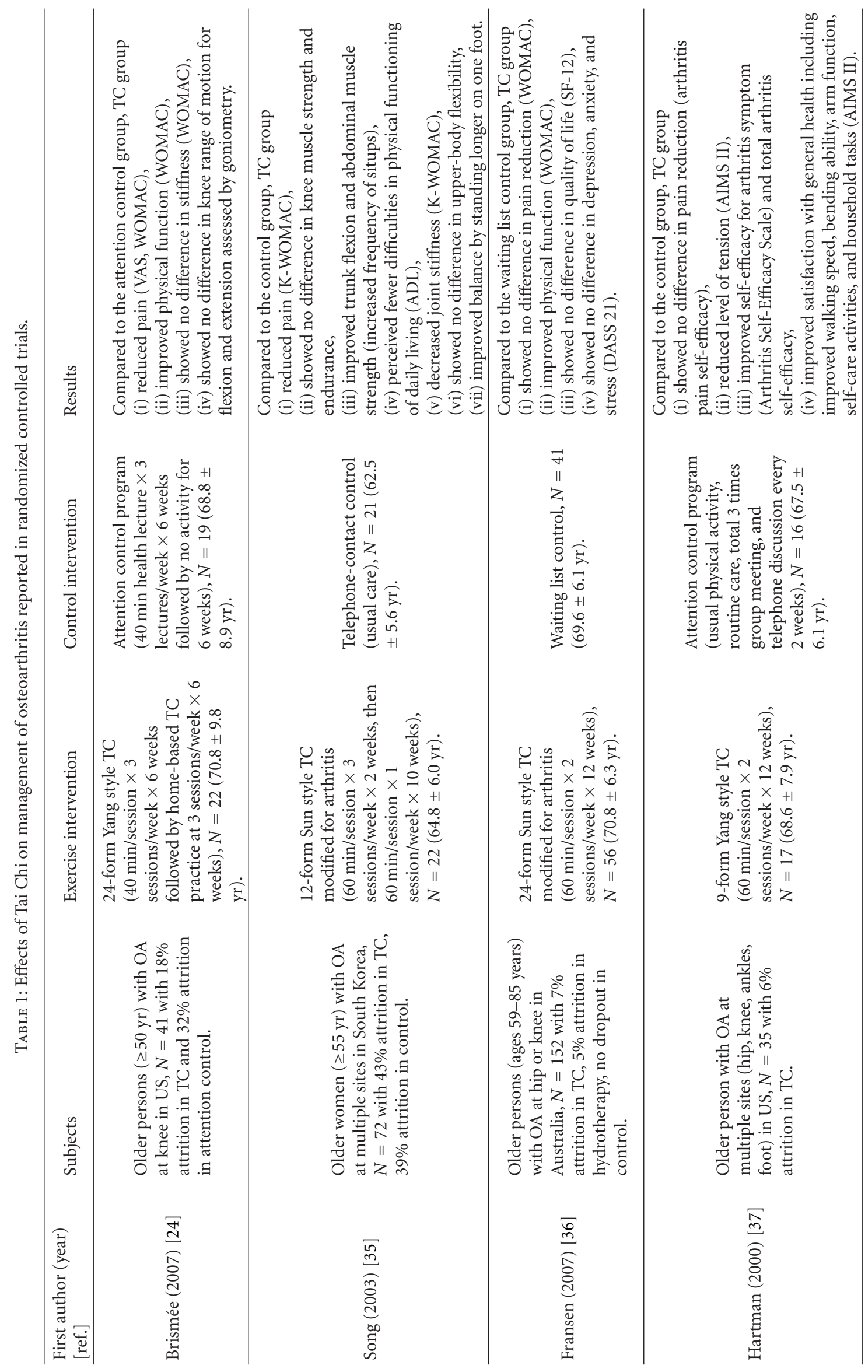




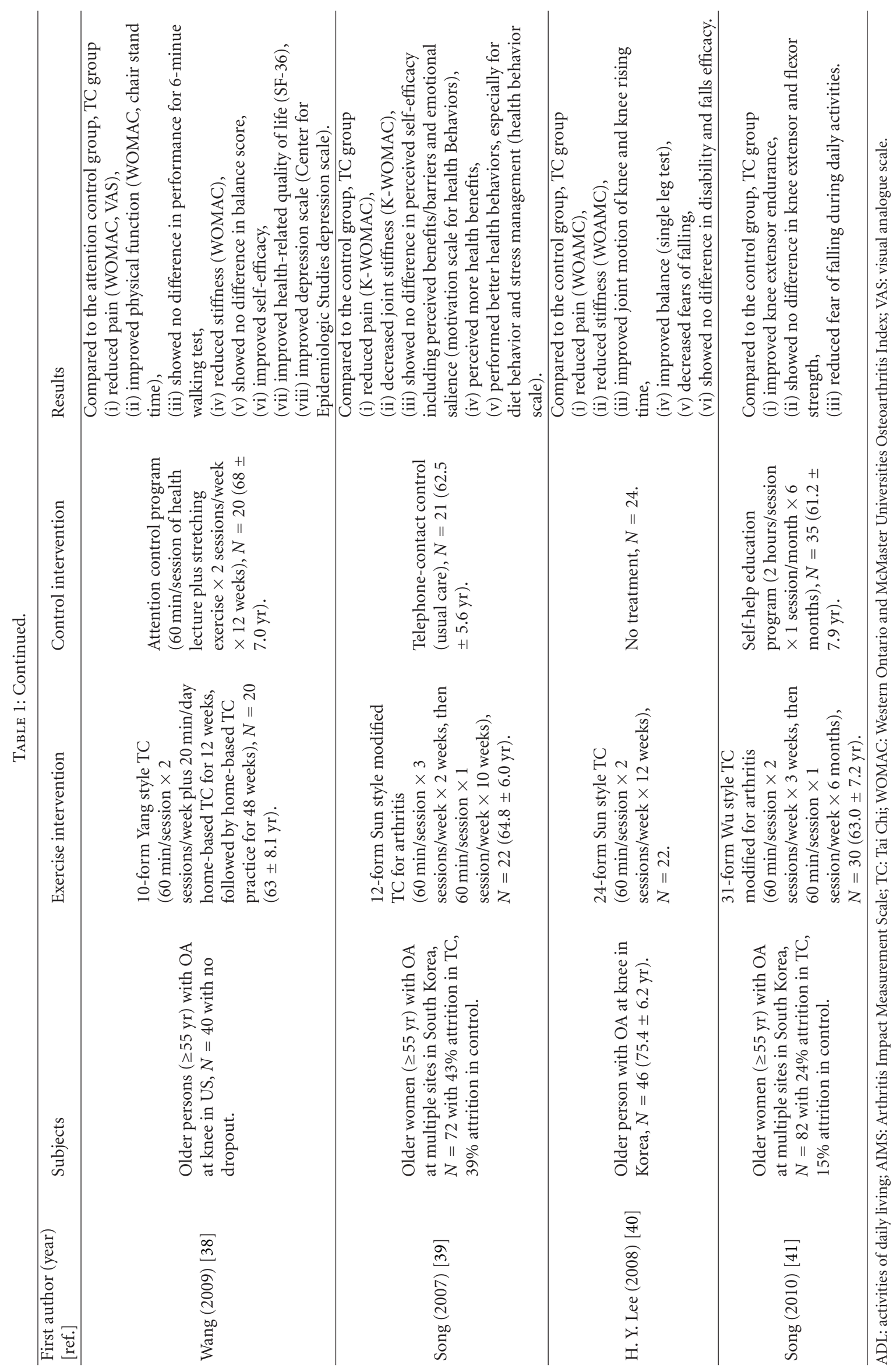


and session length, age, or gender. Ethnicity or geography may play a role, as all studies $[36,37]$ reporting no benefit of TC in OA pain alleviation were conducted outside Asia (Australia and US), while all studies conducted in Korea $[35,39-41]$ as well as some studies conducted in the US $[24,38]$ reported positive outcomes. BMI is usually higher outside Asia, and there is probably a higher expectation of positive outcomes/familiarity of TC in Asian communities compared with Caucasian communities.

2.2. Muscle Strength. Stronger muscles and better coordination improve the stability of the knee joint, resulting in less pain [14]. Thus, muscle strengthening is a key component of exercise in the management of knee OA [14]. Studies have established the beneficial effects of exercise in patients with mild-to-moderate $\mathrm{OA}$ of the knee, including quadriceps muscle strengthening [14].

Only 2 RCTs evaluated TC's effects on muscle strength in OA patients $[35,41]$, both conducted by the same team. Song et al. [35] reported that compared to telephone-contact control (usual care), TC improved the abdominal muscle strength, and trunk flexion (measured by the number of situps performed in 30 seconds) but made no difference in knee muscle strength and endurance [35] (Table 1). More recently, the same research team reported that extending the TC intervention from 12 weeks to 27 weeks resulted in significantly greater knee extensor endurance, while no difference in knee extensor or flexor strength was observed [41]. These two studies suggest that TC may improve abdominal muscle strength, but not knee muscle strength; it may also improve knee extensor endurance after 6 months of intervention.

2.3. Physical Function. Five RCTs investigating TC's effects on physical function in OA patients have been published $[22,35-38]$, and 4 of them reported that TC significantly improves physical function in OA patients (Table 1). TC has been shown to improve physical function based on the results of WOMAC-physical function subscale or activity of daily living compared to waiting list control [36], and usual care treatment [35] in subjects with OA-related symptoms. Brismée et al. [24] reported that a 12-week TC program consisting of 6 weeks of group TC followed by 6 weeks of home-based TC improved physical function (WOMAC physical function-subscale) in the elderly with knee OA, relative to an attention control group. Wang et al. [38] reported that compared to the attention control group, 12week TC intervention improved the physical function, as shown by WOMAC physical function subscale and chair stand time, but TC had no effect on 6-minute walk test performance.

TC was also found to result in no difference compared with hydrotherapy in improving physical function in OA patients [36], suggesting that TC can provide comparable benefits as hydrotherapy in this respect, but TC intervention requires no equipment.

2.4. Stiffness, Range of Motion, and Flexibility. TC movements involve maintaining some degree of knee flexion while moving in various directions. Such pattern of knee movement is associated with larger peak shear forces and larger peak moments in the lower-extremity joints than normal gait [45]. However, the effects of TC on stiffness, range of motion, and flexibility remain inconclusive.

Five RCTs on TC's effects on stiffness, range of motion and flexibility in OA patients have been published [24, 3840] (Table 1). In 4 of these studies, TC significantly reduced joint stiffness compared with control based on WOMACstiffness subscale score $[35,38-40]$, with one of these 4 studies reporting that $\mathrm{TC}$ also improved joint motion of knee and knee rising time [40]. On the other hand, one of the 5 RCTs showed that TC made no difference in upper-body flexibility [35], and another study showed that TC made no difference in either stiffness or knee range of motion [24] compared with attention control. Among these 5 RCTs on TC's effects on stiffness in OA patients, 3 were conducted in Korea and 2 in the US. All studies conducted in Korea demonstrated TC's benefit in reducing joint stiffness [35, 39, 40]. One of the 2 US studies showed TC's positive result in stiffness reduction (Wang et al. [38]). The other US study (Brismée et al. [24]) reported no effect, possibly because its TC intervention period ( 6 weeks of group exercise followed by 6 weeks of home practice) was much shorter compared with that of [38] (12 weeks of group exercise followed by 48 weeks of home practice).

Regarding joint range of motion, H. Y. Lee and K. J. Lee [40] reported that TC improved knee joint motion and rising time relative to control, whereas Brismée et al. [24] found no difference in knee range of motion for flexion and extension between TC group and attention control in subjects with knee OA.

2.5. Balance and Fear of Falling. One of the most effective prevention strategies for reducing the risk of falling is exercise that focuses on improving muscle strength, balance, and coordination $[46,47]$. Women, especially older women with $\mathrm{OA}$, are less likely to participate in any type of physical exercise due to their fear of falling and poor confidence, leading to deconditioning and loss of function [46].

Four RCTs on TC's effects on balance in OA patients have been published $[35,37,38,40]$ (Table 1). In 2 of these studies, TC was shown to significantly improve balance in OA patients compared to usual care [35], and no treatment control [40], measured by one leg standing test. Another study showed that TC tended to improve one-leg balance in OA patients compared to attention control $(P<0.1)$ [37]. However, TC showed no effect on balance relative to attention control in the other study [38] (Wang et al.). Traditional TC involves significant balance training through moves such as the rooster stance featuring one-leg standing, and TC kick featuring standing with one leg while the other leg slowly kicks up and lowers down in a period of about 5 seconds or longer. TC footwork requiring constantly moving the body in different directions in a slow and controlled fashion with one supporting leg also provides remarkable balance training. However, if these balance training moves and elements are not included in the exercise intervention, the beneficial effect of TC on balance may not show. 
Two RCTs on TC's effects on falling in OA patients have been published $[40,41]$ (Table 1). These studies reported that TC decreased fear of falling in OA patients compared to selfhelp education control [41] and no treatment control [40]. On the other hand, the study [40] also showed no impact due to TC on disability and fall efficacy.

2.6. Self-Efficacy. Two RCTs on TC's effects on self-efficacy in OA patients have been published $[37,38]$ (Table 1). These studies reported that TC improved self-efficacy for arthritis symptoms and total arthritis self-efficacy relative to attention control. On the other hand, Song et al. [39] reported no difference in perceived self-efficacy including perceived benefits/barriers and emotional salience, assessed by motivation scale for health behaviors, between TC and usual care.

2.7. Quality of Life. The effect of TC on QOL in OA patients has been evaluated in 3 RCTs [36-38] (Table 1). Among these 3 studies, TC benefited QOL in OA patients in 2 of them, while the other showed no such effect of TC. Hartman et al. [37] reported that TC favored the satisfaction with general health including improved walking speed, bending ability, arm function, self-care activities, and household tasks with arthritis impact measurement scale (AIMS) survey [47]. Wang et al. [38] showed that TC improved health-related QOL measured by SF-36 [49] survey in patients with knee OA. On the other hand, Fransen et al. [36] reported no difference in changes in the physical and mental component summary scores of the SF-12 [50] among TC, hydrotherapy program, and waiting list control.

2.8. Psychological Outcomes. Three RCTs investigating TC's effects on psychological outcomes in OA patients have been published [36, 38, 39], with 2 of them reported positive results (Table 1). Wang et al. reported that TC improved depression symptoms, assessed by Center for Epidemiologic Studies depression scale, compared to attention control [38]. TC made OA patients perceive more health benefits and perform better health behaviors, especially with diet and stress management, assessed by health behavior scale [39]. On the other hand, TC resulted in no difference in depression, anxiety, and stress, measured by DASS 21 , in OA patients [36].

\section{Qigong Exercise for OA Management}

Qigong (Chi Gong or Chi Kung) is an art of moving qi/chi (vital life energy) through the body, releasing energy blocks, and eliminating causes of illness and imbalance. Hundreds of forms of qigong exercises designed for specific or general health enhancement purposes have been created and practiced [51]. According to traditional Chinese medicine, good health is the result of free-flowing, well-balanced qi, while sickness or pain, such as arthritis, is the result of a blockage of the qi flow or unbalanced qi in the body $[42,51,52]$.

Qigong can be divided into internal and external types. Internal qigong refers to individual practices and exercises to achieve optimal dynamic mind-body integration through improved qi circulation such as TC qigong, Baduanjin qigong, and meditation. External qigong involves interaction between a patient and a qigong healer who uses hand movement, acupressure on specific points, focused attention, and/or projection or emission of qi toward the patient's body to improve the flow of qi by breaking qi blockages or removing sick qi, to restore balance of the system, to relieve pain, and/or to cure disease [53-55].

External qigong therapy (EQT) and internal qigong practice may palliate symptoms of arthritis by relaxing diseased tissues and enhancing blood flow to the area [42, 56-58]. Increased blood flow leads to more efficient delivery of oxygen, nutrients, pain-killing substances and drugs, as well as more efficient removal of mediators of pain and metabolic waste products that contribute to pain $[57,58]$. There is considerable variability in the EQTs of different schools and practitioners. Some studies in China reported improvement of severe arthritis symptoms by EQTs [42].

Three RCT studies evaluating the impact of qigong on management of OA have been reported (Table 2). Chen et al. [42] reported that OA subjects in the EQT group, after receiving 5 to 6 sessions of EQT treatment in 3 weeks by two different healers, demonstrated a greater pain reduction and improvement in functionality (as assessed by WOMAC) than those in the placebo-sham group. It was further found that the beneficial effects of EQT on pain reduction and functionality improvement were sustained 3 months after the intervention, but only in subjects treated by one of the 2 healers (healer 2). Subjects treated by this healer also tended to walk faster than the placebo-sham group, but similar result was not observed in those subjects treated by the other healer (healer 1). There was no difference in range of motion, anxiety or depression scale between the EQT group and the sham-control group, regardless of healers. Subjects treated by healer 2 tended to demonstrate lower negative mood level but not in those treated by healer 1. Apparently, the results of the two qigong healers were not consistent, and outcomes of healer 2 seemed to be more beneficial than healer 1, making the results difficult to interpret. This suggests that the effectiveness of EQT may be provider dependent and underscores the difficulty of EQT research as different qigong healer (master) may possess different level of healing qi power that may result in different outcomes in patients. Even the qi power of the same healer may vary depending on his/her own physical condition at the time of treatment. The basic unresolved issue is quantification of the power of qi that is delivered by the healer and actually received by the patient through physical measurements. Before this issue is resolved, EQT clinical studies will continue to be limited by unquantified intervention.

In internal qigong, Baduanjin qigong (translated as the "eight section of brocades", as it contains 8 fine, delicate, and smooth exercise movements) can be learned easily and is less physically and cognitively demanding than TC [43]. Baduanjin was defined as a low-level aerobic exercise that can improve the limbs' range of motion, strength, and general health [43], while it also contains much stretching and controlled breathing aimed to improve circulation of qi. In a pilot feasibility study by An et al. [43], subjects with 
TABLE 2: Effects of Qigong exercise on management of osteoarthritis reported in randomized controlled trials.

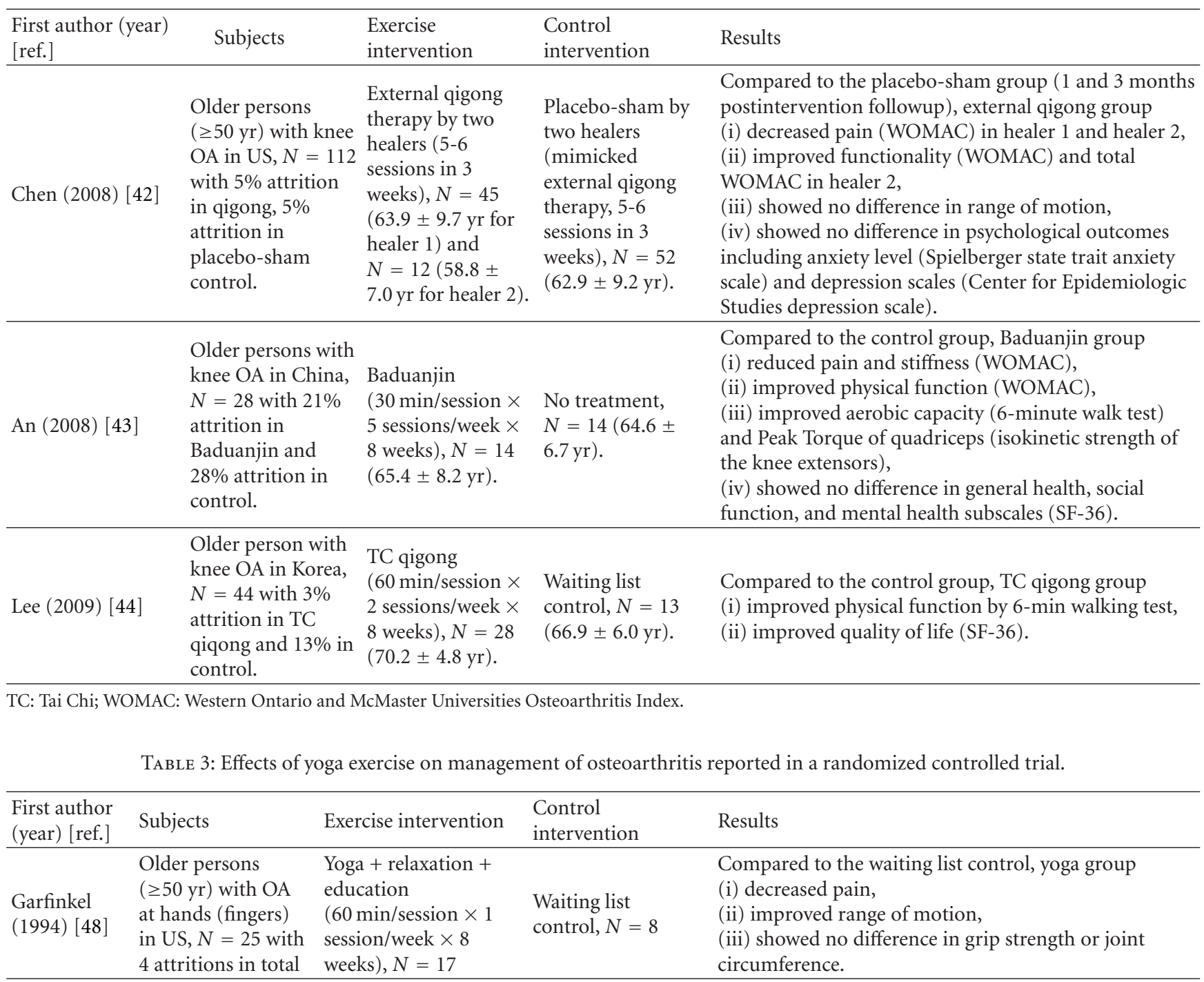

OA reported reduced knee pain and stiffness, and improved physical function in the Baduanjin group compared to the control group (Table 2). In addition, subjects in the Baduanjin group demonstrated statistically significant improvement in aerobic capacity (assessed by 6-minute walking test) as well as quadriceps strength (assessed by isokinetic strength of the knee extensors) [43]. However, there was no difference in SF-36 between the Baduanjin group and the control group (Table 2).

TC qigong, another form of internal qigong, is a combination of TC movement with qigong training including incorporating intention into movement and enhancing awareness of breathing [44]. Basically, TC qigong can be considered as TC focusing on qi and without martial applications. The TC qigong implemented in [44] consisted of a sequence of 18 movements, performed six times in a fluid and continuous manner, combined with deep abdominal breathing. It was claimed that TC qigong is an easy and safe qigong for patients, because it is simpler and more repetitive than TC, and it is especially suitable for patients with joint problems, as the motion does not impose undue pressure on the pivot joint, such as the waist, knee, and ankles during training [44]. TC qigong improved physical function (measured by 6-min walking test) and quality of life (measured by SF-36) compared to the waiting list control [44].

\section{Yoga Exercise for OA Management}

Yoga is a CAM mind-body practice, with origins in ancient Indian philosophy, that combines physical postures, breathing techniques, and meditation or relaxation. A 2008 survey of 5,050 adults indicated that $6.9 \%$ of U.S. adults, or 15.8 million people, practice yoga [59]. Among numerous styles of yoga, Hatha yoga is the most commonly practiced in the United States and Europe. Hatha yoga was originally designed as a preparatory practice for physical purification before meditation and has been adopted in the Western civilization as a therapy to increase flexibility, strength, and stamina, while also promoting self-awareness, emotional 
stability, and peace of mind. Yoga implements controlled postures, breathing and meditation to achieve these goals $[60,61]$. It usually involves holding the body in a sequence of postures for a certain period of time, breathing exercises, and meditation.

The practice of Hatha yoga has been shown to reduce pain, relieve tension, reduce risks of injury, improve posture, improve communication, increase energy and attention span, and enhance feelings of overall wellness and well-being [62]. The use of Iyengar approach to Hatha yoga emphasizes strength, flexibility, and relaxation, with particular attention to alignment of body structures (e.g., the relationship of the distal to the proximal extremities and the extremities to the spine and torso) $[48,63,64]$. Raman suggested that lyengar method of Hatha yoga can be used as a supplement to other measures to treat musculoskeletal problems [65]. Yoga is one of several practices that have the potential to be beneficial to OA $[66,67]$.

There is only one RCT study published evaluating the impact of yoga on management of OA (Table 3). In their RCT of patients with hand OA, Garfinkel et al. showed that yoga exercise significantly decreased finger pain during activity, improved finger range of motion, but showed no difference in grip strength or joint circumference compared to the waiting list control [66].

\section{Conclusion and Future Research}

Findings based on only a small number of RCTs available in the literature suggest that CAM exercises-Tai Chi, qigong, and yoga-demonstrate considerable promise in the management of OA symptoms. In particular, it has been shown that TC may reduce arthritic pain, enhance physical functioning and flexibility, and improve self-efficacy and quality of life in OA patients. However, most existing studies suffer methodological limitations (e.g., small sample size, nonrandomized trial, inconsistent intervention, short intervention duration, inconsistent instruments for outcomes assessment, and short follow-up periods) that hinder interpretation of findings and preclude firm conclusions. Further, there may be reasons for false positive results, such as participants unblinded to allocation, Hawthorne effect multiple outcomes measured. Future studies require rigorous and high-quality randomized controlled trials with larger sample sizes along with standardized and quantified intervention and quantitative assessments for pain reduction, muscle strength and endurance, physical performance, joint motion and stiffness, flexibility, and OA-related biofluid biomarkers that have been shown to be reliable and responsive to change due to exercises.

\section{References}

[1] N. Arden and M. C. Nevitt, "Osteoarthritis: epidemiology," Best Practice \& Research: Clinical Rheumatology, vol. 20, no. 1, pp. 3-25, 2006.

[2] S. K. Das and A. Farooqi, "Osteoarthritis," Best Practice \& Research: Clinical Rheumatology, vol. 22, no. 4, pp. 657-675, 2008.
[3] World Health Organization, "Chronic rheumatic conditions," January 2011, http://www.who.int/chp/topics/rheumatic/en/print.html.

[4] R. Bitton, "The economic burden of osteoarthritis," The American Journal of Managed Care, vol. 15, no. 8, pp. S230S235, 2009.

[5] E. M. Roos, W. Herzog, J. A. Block, and K. L. Bennell, "Muscle weakness, afferent sensory dysfunction and exercise in knee osteoarthritis," Nature Reviews Rheumatology, vol. 7, no. 1, pp. 57-63, 2010.

[6] M. E. Tinetti, C. Gordon, E. Sogolow, P. Lapin, and E. H. Bradley, "Fall-risk evaluation and management: challenges in adopting geriatric care practices," Gerontologist, vol. 46, no. 6 , pp. 717-725, 2006.

[7] G. Jones, T. Nguyen, P. N. Sambrook, S. R. Lord, P. J. Kelly, and J. A. Eisman, "Osteoarthritis, bone density, postural stability, and osteoporotic fractures: a population based study," Journal of Rheumatology, vol. 22, no. 5, pp. 921-925, 1995.

[8] E. K. Wiley-Exley, T. J. Mielenz, E. C. Norton, and L. F. Callahan, "Complementary and alternative medicine use in musculoskeletal disorders: does medical skepticism matter?" Open Rheumatology Journal, vol. 1, no. 1, pp. 5-11, 2007.

[9] M. A. Fitzcharles, D. Lussier, and Y. Shir, "Management of chronic arthritis pain in the elderly," Drugs \& Aging, vol. 27, no. 6, pp. 471-490, 2010.

[10] S. Y. Shin and A. M. Kolanowski, "Best evidence of psychosocially focused nonpharmacologic therapies for symptom management in older adults with osteoarthritis," Pain Management Nursing, vol. 11, no. 4, pp. 234-244, 2010.

[11] G. A. Hawker, S. Mian, K. Bednis, and I. Stanaitis, "Osteoarthritis year 2010 in review: non-pharmacologic therapy," Osteoarthritis and Cartilage, vol. 19, no. 4, pp. 366-374, 2011.

[12] K. Burks, "Osteoarthritis in older adults: current treatments," Journal of Gerontological Nursing, vol. 31, no. 5, pp. 11-1960, 2005.

[13] C. Wang, "Tai Chi and rheumatic diseases," Rheumatic Disease Clinics of North America, vol. 37, no. 1, pp. 19-32, 2011.

[14] W. Zhang, R. W. Moskowitz, G. Nuki et al., "OARSI recommendations for the management of hip and knee osteoarthritis, Part II: OARSI evidence-based, expert consensus guidelines," Osteoarthritis and Cartilage, vol. 16, no. 2, pp. 137-162, 2008.

[15] S. M. Bertisch, C. C. Wee, R. S. Phillips, and E. P. McCarthy, "Alternative mind-body therapies used by adults with medical conditions," Journal of Psychosomatic Research, vol. 66, no. 6, pp. 511-519, 2009.

[16] M. V. Hurley, "Quadriceps weakness in osteoarthritis," Current Opinion in Rheumatology, vol. 10, no. 3, pp. 246-250, 1998.

[17] M. V. Hurley, D. L. Scott, J. Rees, and D. J. Newham, "Sensorimotor changes and functional performance in patients with knee osteoarthritis," Annals of the Rheumatic Diseases, vol. 56, no. 11, pp. 641-648, 1997.

[18] G. D. Deyle, N. E. Henderson, R. L. Matekel, M. G. Ryder, M. B. Garber, and S. C. Allison, "Effectiveness of manual physical therapy and exercise in osteoarthritis of the knee: a randomized, controlled trial," Annals of Internal Medicine, vol. 132, no. 3, pp. 173-181, 2000.

[19] M. E. Van Baar, J. Dekker, J. A. M. Lemmens, R. A. B. Oostendorp, and J. W. J. Bijlsma, "Pain and disability in patients with osteoarthritis of hip or knee: the relationship with articular, kinesiological, and psychological characteristics," Journal of Rheumatology, vol. 25, no. 1, pp. 125-133, 1998. 
[20] B. T. Maurer, A. G. Stern, B. Kinossian, K. D. Cook, and H. R. Schumacher, "Osteoarthritis of the knee: isokinetic quadriceps exercise versus and educational intervention," Archives of Physical Medicine and Rehabilitation, vol. 80, no. 10, pp. 12931299, 1999.

[21] P. M. Barnes, B. Bloom, and R. L. Nahin, "Complementary and alternative medicine use among adults and children: United States, 2007," National Health Statistics Reports, no. 12, pp. 123, 2009.

[22] L. Suen, "The role of complementary therapies in cardiac care: where are we now?" British Journal of Cardiac Nursing, vol. 2, pp. 80-85, 2007.

[23] G. S. Birdee, P. M. Wayne, R. B. Davis, R. S. Phillips, and G. Y. Yeh, "T'ai Chi and Qigong for health: patterns of use in the united states," Journal of Alternative and Complementary Medicine, vol. 15, no. 9, pp. 969-973, 2009.

[24] J. M. Brismée, R. L. Paige, M. C. Chyu et al., "Group and home-based tai chi in elderly subjects with knee osteoarthritis: a randomized controlled trial," Clinical Rehabilitation, vol. 21, no. 2, pp. 99-111, 2007.

[25] K. L. Bennell and R. S. Hinman, "A review of the clinical evidence for exercise in osteoarthritis of the hip and knee," Journal of Science and Medicine in Sport, vol. 14, no. 1, pp. 4-9, 2011.

[26] C. Lan, J. S. Lai, and S. Y. Chen, "Tai Chi Chuan: an ancient wisdom on exercise and health promotion," Sports Medicine, vol. 32, no. 4, pp. 217-224, 2002.

[27] A. M. Kuramoto, "Therapeutic benefits of Tai Chi exercise: research review," Wisconsin Medical Journal, vol. 105, no. 7, pp. 42-46, 2006.

[28] P. A. Adler and B. L. Roberts, "The use of Tai Chi to improve health in older adults," Orthopaedic Nursing, vol. 25, no. 2, pp. 122-126, 2006.

[29] C. Wang, J. P. Collet, and J. Lau, "The effect of Tai Chi on health outcomes in patients with chronic conditions: a systematic review," Archives of Internal Medicine, vol. 164, no. 5, pp. 493-501, 2004.

[30] P. F. Tsai, C. Beck, J. Y. Chang et al., "The effect of Tai Chi on knee osteoarthritis pain in cognitively impaired elders: pilot study," Geriatric Nursing, vol. 30, no. 2, pp. 132-139, 2009.

[31] Y. Escalante, A. García-Hermoso, and J. M. Saavedra, "Effects of exercise on functional aerobic capacity in lower limb osteoarthritis: a systematic review," Journal of Science and Medicine in Sport, 2010.

[32] Y. Escalante, J. M. Saavedra, A. García-Hermoso, A. J. Silva, and T. M. Barbosa, "Physical exercise and reduction of pain in adults with lower limb osteoarthritis: a systematic review," Journal of Back and Musculoskeletal Rehabilitation, vol. 23, no. 4, pp. 175-186, 2010.

[33] P. A. Adler, The effects of tai chi on pain and function in older adults with osteoarthritis, Ph.D. dissertation, Frances Payne Bolton School of Nursing. Case Western Reserve University, Cleveland, Ohio, USA, 2007.

[34] P. Adler, M. Good, B. Roberts, and S. Snyder, "The effects of Tai Chi on older adults with chronic arthritis pain," Journal of Nursing Scholarship, vol. 32, no. 4, p. 377, 2000.

[35] R. Song, E. O. Lee, P. Lam, and S. C. Bae, "Effects of tai chi exercise on pain, balance, muscle strength, and perceived difficulties in physical functioning in older women with osteoarthritis: a randomized clinical trial," Journal of Rheumatology, vol. 30, no. 9, pp. 2039-2044, 2003.
[36] M. Fransen, L. Nairn, J. Winstanley, P. Lam, and J. Edmonds, "Physical activity for osteoarthritis management: a randomized controlled clinical trial evaluating hydrotherapy or Tai Chi classes," Arthritis Care \& Research, vol. 57, no. 3, pp. 407414, 2007.

[37] C. A. Hartman, T. M. Manos, C. Winter, D. M. Hartman, B. Li, and J. C. Smith, "Effects of T'i Chi training on function and quality of life indicators in older adults with osteoarthritis," Journal of the American Geriatrics Society, vol. 48, no. 12, pp. 1553-1559, 2000.

[38] C. Wang, C. H. Schmid, P. L. Hibberd et al., "Tai Chi is effective in treating knee osteoarthritis: a randomized controlled trial," Arthritis Care \& Research, vol. 61, no. 11, pp. 1545-1553, 2009.

[39] R. Song, E. O. Lee, P. Lam, and S. C. Bae, "Effects of a Sun-style Tai Chi exercise on arthritic symptoms, motivation and the performance of health behaviors in women with osteoarthritis," Journal of Korean Academy of Nursing, vol. 37, no. 2, pp. 249-256, 2007.

[40] H. Y. Lee and K. J. Lee, "Effects of tai chi exercise in elderly with knee osteoarthritis," Journal of Korean Academy of Nursing, vol. 38, no. 1, pp. 11-18, 2008.

[41] R. Song, B. L. Roberts, E. O. Lee, P. Lam, and S. C. Bae, "A randomized study of the effects of t'ai chi on muscle strength, bone mineral density, and fear of falling in women with osteoarthritis," Journal of Alternative and Complementary Medicine, vol. 16, no. 3, pp. 227-233, 2010.

[42] K. W. Chen, A. Perlman, J. G. Liao, A. Lam, J. Staller, and L. H. Sigal, "Effects of external qigong therapy on osteoarthritis of the knee," Clinical Rheumatology, vol. 27, no. 12, pp. 14971505, 2008.

[43] B. An, K. Dai, Z. Zhu et al., "Baduanjin alleviates the symptoms of knee osteoarthritis," Journal of Alternative and Complementary Medicine, vol. 14, no. 2, pp. 167-174, 2008.

[44] H. J. Lee, H. J. Park, Y. Chae et al., "Tai Chi Qigong for the quality of life of patients with knee osteoarthritis: a pilot, randomized, waiting list controlled trial," Clinical Rehabilitation, vol. 23, no. 6, pp. 504-511, 2009.

[45] G. Wu and D. Millon, "Joint kinetics during Tai Chi gait and normal walking gait in young and elderly Tai Chi Chuan practitioners," Clinical Biomechanics, vol. 23, no. 6, pp. 787795, 2008.

[46] B. J. Messinger-Rapport and H. L. Thacker, "Prevention for the older woman: mobility: a practical guide to managing osteoarthritis and falls. Part 6," Geriatrics, vol. 58, no. 7, pp. 22-29, 2003.

[47] R. F. Meenan, J. H. Mason, J. J. Anderson, A. A. Guccione, and L. E. Kazis, "AIMS2: the content and properties of a revised and expanded arthritis impact measurement scales health status questionnaire," Arthritis and Rheumatism, vol. 35, no. 1, pp. 1-10, 1992.

[48] M. S. Garfinkel, H. R. Schumacher, A. Husain, M. Levy, and R. A. Reshetar, "Evaluation of a yoga based regimen for treatment of osteoarthritis of the hands," Journal of Rheumatology, vol. 21, no. 12, pp. 2341-2343, 1994.

[49] J. Ware Jr., K. K. Snow, M. Kosinski, and B. Gandek, SF36 Health Survey Manual and Interpretation Guide, Health Institute, New England Medical Center Hospital, Boston, Mass, USA, 1993.

[50] B. Gandek, J. E. Ware, N. K. Aaronson et al., "Cross-validation of item selection and scoring for the SF-12 Health Survey in nine countries: results from the IQOLA Project," Journal of Clinical Epidemiology, vol. 51, no. 11, pp. 1171-1178, 1998. 
[51] T. J. Liu, Ed., Qigong Studies in Chinese Medicine (Textbook Used in Universities and Colleges in China), China Publisher of Chinese Medicine, Beijing, China, 2005.

[52] Z. S. Weng, "60 cases of rheumatoid arthritis in late stage treated by medicine fumigation and washing combined with Qi-conducting pressing acupoints and Tui Na," China's Naturopathy, vol. 10, no. 5, p. 12, 1998.

[53] K. M. Sancier, "Medical applications of qigong," Alternative Therapies in Health and Medicine, vol. 2, no. 1, pp. 40-46, 1996.

[54] M. S. Lee, M. H. Pittler, and E. Ernst, "External qigong for pain conditions: a systematic review of randomized clinical trials," Journal of Pain, vol. 8, no. 11, pp. 827-831, 2007.

[55] A. Vincent, J. Hill, K. M. Kruk, S. S. Cha, and B. A. Bauer, "External qigong for chronic pain," American Journal of Chinese Medicine, vol. 38, no. 4, pp. 695-703, 2010.

[56] Y. Machi and C. Liu, "Measurements of physiological effects of internal qigong: the six-word practice," Journal of International Society of Life Information Science, vol. 17, no. 1, pp. 32-47, 1999.

[57] Y. Xiaohai and D. Songxiang, "Rheumatoid arthritis treated by qigong combined with herb meals," Practical Journal of Integrating Chinese with Modern Medicine, vol. 10, no. 15, p. 1506, 1997.

[58] G. Litscher, G. Wenzel, G. Niederwieser, and G. Schwarz, "Effects of QiGong on brain function," Neurological Research, vol. 23, no. 5, pp. 501-505, 2001.

[59] "Yoga in America Study," January 2011, http://www.yogajour. nal.com/press/yoga_in_america.

[60] M. Garfinkel and H. R. Schumacher Jr., "Yoga," Rheumatic Disease Clinics of North America, vol. 26, no. 1, pp. 125-132, 2000.

[61] E. Y. Hanada, "Efficacy of rehabilitative therapy in regional musculoskeletal conditions," Best Practice \& Research: Clinical Rheumatology, vol. 17, no. 1, pp. 151-166, 2003.

[62] S. T. Gura, "Yoga for stress reduction and injury prevention at work," Work, vol. 19, no. 1, pp. 3-7, 2002.

[63] B. K. S. Iyengar, Light on Yoga. Part II, Schocken Books, New York, NY, USA, 1979.

[64] B. K. S. Iyengar, The Tree of Yoga, Fine Line Books, Oxford, UK, 1998.

[65] K. Raman, "A matter of health," in Integration of Yoga and Western Medicine for Prevention and Cure, Eastwest Books, Chennai, India, 1998.

[66] S. L. Kolasinski, "Yoga for degenative joint disease," Alternative Medicine Alert, vol. 4, no. 1, pp. 28-31, 2001.

[67] S. L. Kolasinski, "The use of alternative therapies by patients with rheumatic diseases," Journal of Clinical Rheumatology, vol. 5, no. 5, pp. 253-254, 1999. 


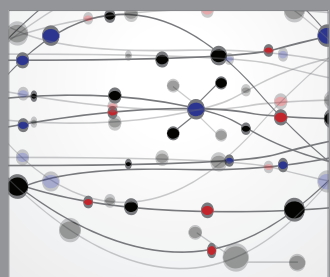

The Scientific World Journal
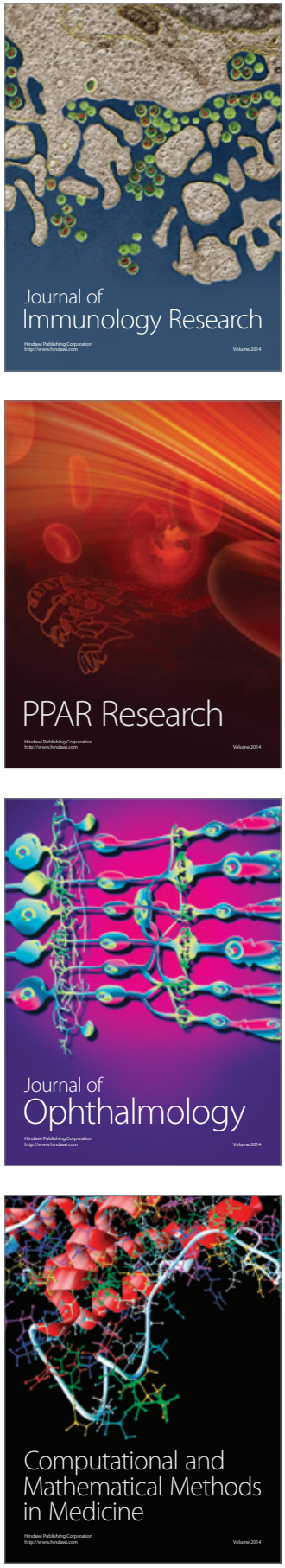

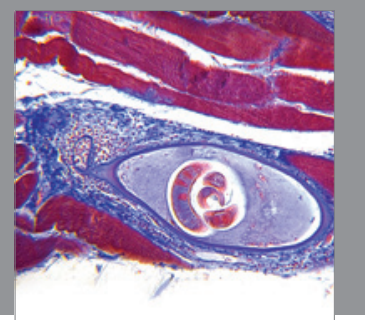

Gastroenterology

Research and Practice
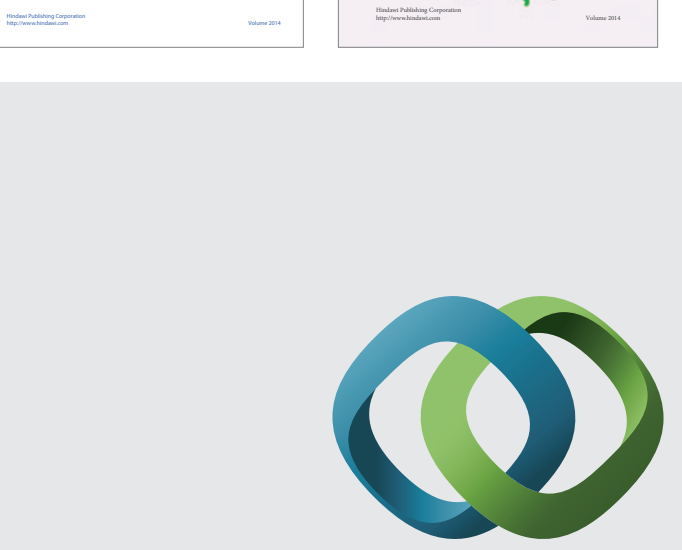

\section{Hindawi}

Submit your manuscripts at

http://www.hindawi.com
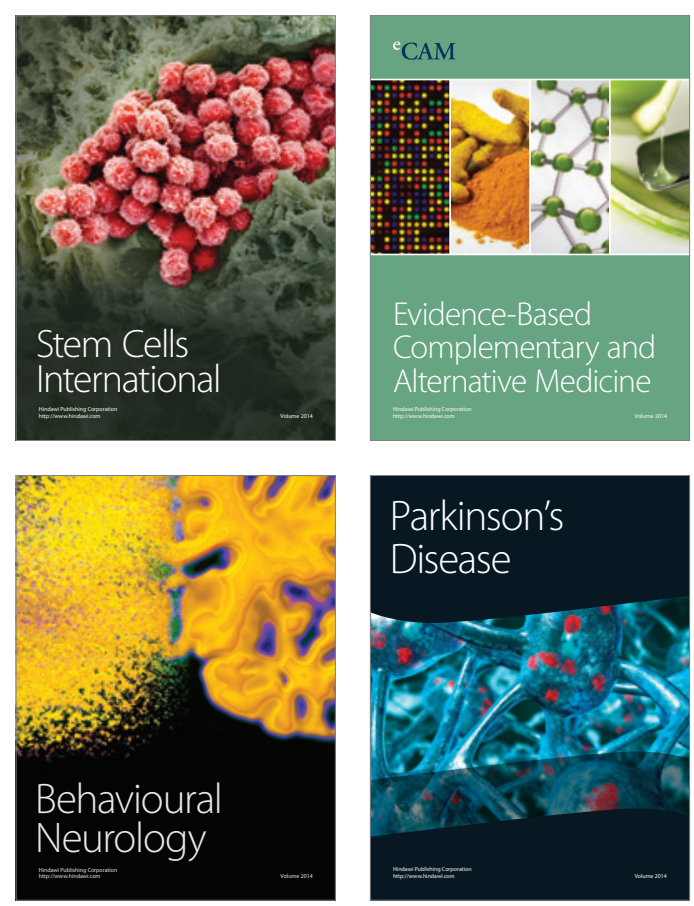

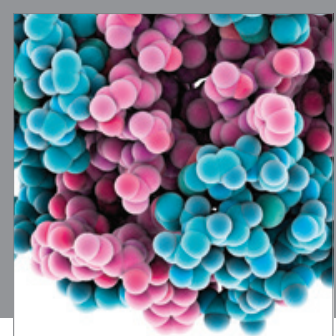

Journal of
Diabetes Research

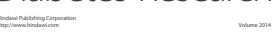

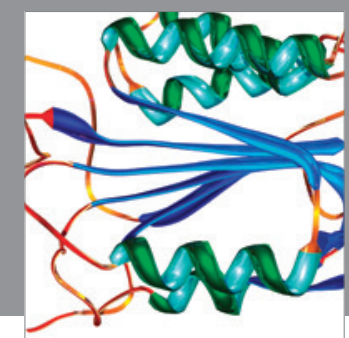

Disease Markers
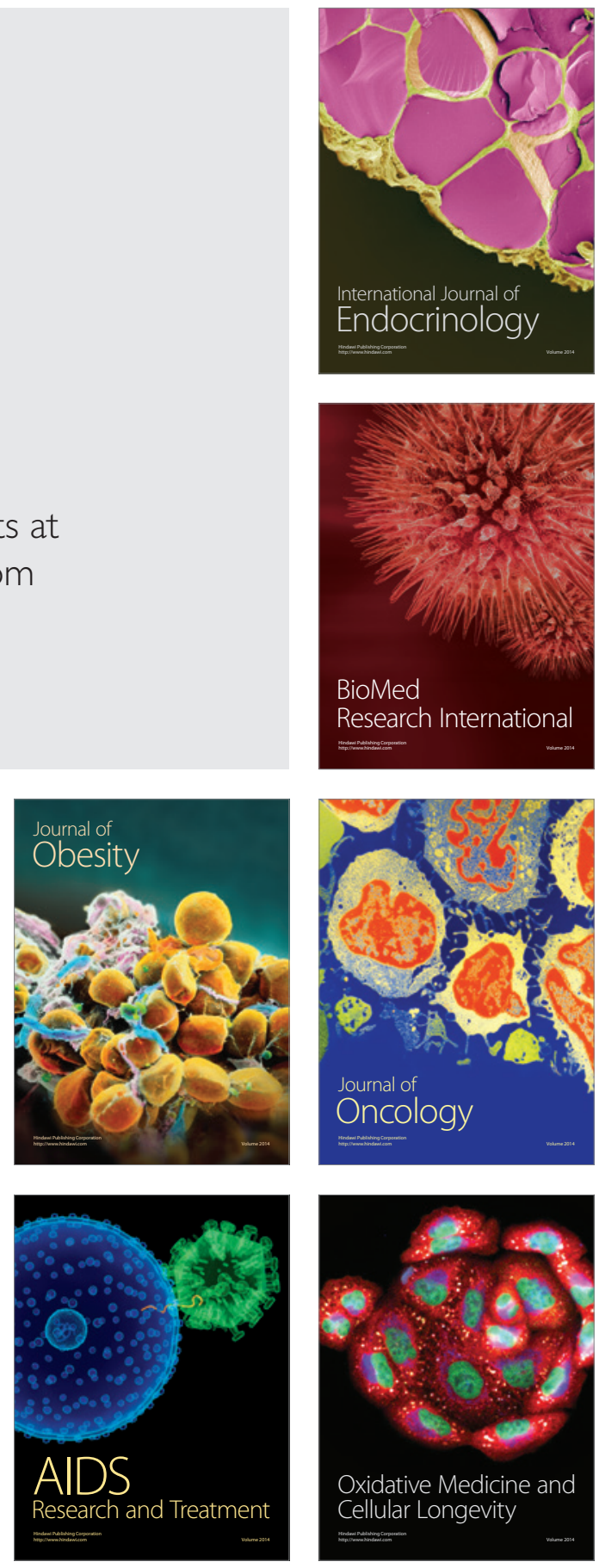